\title{
Potencia aprovechable y variabilidad del viento caso típico distrito de Pimentel
}

\section{Available power and wind variability typical case of the Pimentel district}

\author{
José Pérez1, José Arriola', Max Espinal'1 \\ ${ }^{1}$ Facultad de Ingeniería Mecánica y Eléctrica, Universidad Nacional Pedro Ruiz Gallo, \\ Lambayeque-Perú
}

Recibido : 30/05/2017 Aceptado: 14/08/2017

\section{RESUMEN}

El presente artículo inicia dando a conocer las variables meteorológicas de un parque eólico. Posteriormente se analizan las ecuaciones que determinan la ley de Betz y la distribución Weibull esto con el fin de comprender la cantidad de energía y horas aprovechadas por un aerogenerador, se continúa con el factor de carga de un parque eólico. Finalmente se muestra la influencia de la rugosidad del terreno en la variación del viento y la selección de la zona de emplazamiento.

Palabras Claves: Potencial eólico, Ley de Betz, Distribución de Weibull, factor de carga, rugosidad

\section{ABSTRACT}

The present work begins by describing the meteorological variables of a wind farm. Subsequently, the equations defining Betz's law and the Weibull distribution are analyzed, in order to understand the amount of power and time of operation available from a wind turbine, as well as the load factor of a wind farm. Finally, the influence of surface roughness on wind variation and the selection of a proper location are discussed.

Keywords: wind power, Betz's law, Weibull distribution, load factor, surface roughness

\section{INTRODUCCIÓN}

El aprovechamiento de las energías renovables en el Perú en los últimos años ha venido teniendo un gran impulso, tal es así que el 13 de septiembre del 2010 mediante el Decreto Legislativo $\mathrm{N}^{\circ} 1002$ se promueve el desarrollo de la Generación Eléctrica con Recursos Energéticos Renovables (RER), mediante subastas de energías renovables (procesos supervisados por OSINERGMIN y dirigidos por el MINEM. Esto con el fin de reducir el impacto ambiental, evitando el uso de combustibles fósiles [1].

Los estudios conocidos desarrollados para aprovechar energéticamente las fuentes renovables en el departamento de Lambayeque son: los realizados para la electrificación rural masiva mediante sistemas fotovoltaicos los cuales están incluidos en el Plan Nacional de Electrificación Rural desarrollado por la Dirección General de Electrificación Rural (2013-2022) [2]. Además, se encuentra el estudio de factibilidad de la Central Eólica José Quiñones, actualmente en desarrollo por la empresa Auster Energy desde mayo del 2015 [3].

Según el COES-SINAC en su "Estudio de la máxima capacidad de generación no convencional (eólica y solar) a ser instalada en el SEIN (CMGNC)" [4] el porcentaje de la demanda cubierta en el año 2018 por recursos renovables (solar y eólico) será el $6.4 \%$.

Tabla 1: "Demanda total y máxima capacidad nueva RER esperada al 2018". Fuente: [4]

\begin{tabular}{|c|c|c|c|c|}
\hline $\begin{array}{c}\text { Demanda SEIN esperada } \\
\text { al } 2018\end{array}$ & \multicolumn{3}{|c|}{$\begin{array}{r}\text { Máxima capacidad nueva rer (solar + } \\
\text { eolico) en el SEIN al } 2018\end{array}$} \\
\hline $\begin{array}{c}\text { Pico } \\
{[\mathrm{MW}]}\end{array}$ & $\begin{array}{c}\text { Valor Medio } \\
\text { GWh/año }\end{array}$ & MW & $\begin{array}{c}\text { Valor Medio } \\
\text { GWh/año }\end{array}$ & $\begin{array}{c}\text { respecto } \\
\text { a la } \\
\text { demanda } \\
\text { esperada }\end{array}$ \\
\hline 7450.0 & 59790.0 & 1656.0 & 3786.2 & 6.4 \\
\hline
\end{tabular}

\footnotetext{
* Correspondencia:

E-mail: jcpsalazar17@hotmail.com, jose.cancer2008@hotmail.com, max_07_12@hotmail.com
} 


\subsection{PLANTEAMIENTO DE OBJETIVOS}

- Conocer las variables necesarias para determinar el potencial eólico de una zona específica.

- Analizar la ley de Betz.

- Analizar la distribución de Weibull.

- Analizar el factor de carga de un parque eólico.

- Mostrar la importancia de la rugosidad para la viabilidad

\subsection{PLANTEAMIENTO DE HIPÓTESIS}

La velocidad, la rugosidad del terreno, y la densidad del aire condicionan el aprovechamiento energético de una determinada zona.

\section{ENERGÍA PRESENTE EN EL VIENTO}

Según [6] la energía cinética de una masa de aire $m$ moviéndose con una velocidad $\mathrm{V}$ es obtenida de la siguiente ecuación:

$$
E_{c}=\frac{1}{2} m V^{2}
$$

Ecuación 1: "Energía cinética aprovechable"

El flujo de aire $M$ se expresa como sigue a continuación:

$$
\begin{gathered}
M=\rho A V \\
\text { Ecuación 2: "Flujo de aire" }
\end{gathered}
$$

Dónde:

- M: flujo de aire en $(\mathrm{kg} / \mathrm{s})+$

- p: densidad volumétrica del aire $(\mathrm{kg} / \mathrm{m} 3)$

- A: área de barrido de las palas del rotor (m2)

- V: velocidad del viento $(\mathrm{m} / \mathrm{s})$

Ahora derivando la ecuación 1 con respecto del tiempo $(\mathrm{t})$ se obtiene la potencia $(\mathrm{Pc})$ debida a la energía cinética por la que la ecuación que describe el fenómeno queda definida por [6]:

$$
\frac{d\left(E_{c}\right)}{d t}=P_{c}=\frac{1}{2}\left(\frac{d(m)}{d t}\right) V^{2}=\frac{1}{2} M V^{2}
$$

Ecuación 3: "Energía cinética derivada respecto del tiempo"

Sustituyendo la ecuación 2 en la 3 la potencia cinética queda expresa así:

$$
P_{c}=\frac{1}{2}(\rho A V) V^{2}=\frac{1}{2} \rho A V^{3}
$$

Ecuación 4: "Potencia cinética aprovechable"

\section{DENSIDAD DE POTENCIA DISPONIBLE DEL VIENTO}

El recurso eólico en una zona determinada puede calcularse de manera precisa por la velocidad media del viento, pero la densidad de potencia disponible en el viento proporciona una indicación más correcta del potencial de energía eólica. La densidad de potencial disponible en el viento expresa la energía eólica contenida por metro cuadrado $(\mathrm{W} / \mathrm{m} 2)$ y se obtiene por la siguiente ecuación [6]:

$$
W P D=\frac{1}{2 n} \sum_{i=1}^{n} \rho V i^{3}
$$

Ecuación 5: "Densidad de potencia del viento aprovechable"

Dónde:

- WPD: densidad de potencia del viento (W/m2)

- N: número de registros en el intervalo utilizado para el promedio

- p: densidad del viento $(\mathrm{kg} / \mathrm{m} 3)$ en una hora particular de observación

- Viz: el cubo de la velocidad del viento $(\mathrm{m} / \mathrm{s})$ a la misma hora de observación

El uso de esta última ecuación solo debe ser para registros individuales de medición (por hora, cada diez minutos, etc.) y no para registros promedios a largo plazo como son el valor mensual o anual.

Como se sabe la duración de la densidad del viento depende de la temperatura $(T)$ y de la presión $(P)$. Por tanto si se conoce estas dos variables ( $T$ y $P$ ), la densidad del viento se calcula por la siguiente ecuación:

$$
\rho=\frac{P}{R T}
$$

Ecuación 6: "Densidad del viento con T y P variando"

Dónde:

- p: densidad del viento $(\mathrm{Kg} / \mathrm{m} 3)$

- P: presión del aire (N/m2)

- R: constante especifica de gas del aire (287 J/ Kg.K)

- T: temperatura del aire en grados Kelvin $\left(C^{\circ}+273\right)$

Si la presión del sitio no está disponible, la densidad del puede estimarse como una función de la elevación del sitio y la temperatura de la siguiente manera:

$$
\rho=\frac{P_{0}}{R T} e^{\left(\frac{-g z}{R T}\right)}
$$

Ecuación 7: “Densidad del viento con en función de la elevación y la temperatura" 
Dónde:

- p: densidad del viento ( $\mathrm{Kg} / \mathrm{m} 3)$

- Po: presión atmosférica estándar a nivel del mar (101.325 Pa)

- g: constante gravitacional $(9.8 \mathrm{~m} / \mathrm{s} 2)$

- z: elevación del sitio sobre el nivel del mar (m).

\section{MÁXIMA POTENCIA POSIBLE A EXTRAER DEL VIENTO}

La potencia real a extraer del viento por las palas del rotor es la diferencia entre la energía río arriba y río abajo. Utilizando la ecuación (3) se obtiene:

$$
P_{c}=\frac{1}{2} M\left(V^{2}-V_{0}^{2}\right)
$$

Ecuación 8: "Potencial aprovechable real"

Dónde:

- Pc: potencia mecánica extraída por el rotor.

- V: velocidad del viento río arriba a la entrada de las palas del rotor.

- Vo: velocidad del viento río abajo a la salida de las palas del rotor.

El flujo de aire a través de las palas es, por lo tanto; el obtenido por la multiplicación de la densidad con la velocidad promedio, y queda así:

$$
M=\rho A\left(\frac{V+V_{0}}{2}\right)
$$

Ecuación 9: “Flujo del aire considerando velocidades del viento río arriba y abajo"

Sustituyendo la ecuación (8) en (7) se calcula la potencia mecánica extraída por las palas del rotor que es conducida al generador eléctrico:

$$
P_{c}=\frac{1}{2}\left[\rho A \frac{V+V_{0}}{2}\right]\left(V^{2}-V_{0}^{2}\right)
$$

Reordenando la expresión tenemos:

$$
P_{c}=\frac{1}{2} \rho A V^{3} C_{p}
$$

Obteniéndose:

$$
C_{p}=\frac{\left(1+\frac{V_{0}}{V}\right)\left[1-\left(\frac{V_{0}}{V}\right)^{2}\right]}{2}
$$

Ecuación 10: “Coeficiente de potencia o eficiencia del rotor"
Donde $\mathrm{Cp}$ es la fracción de energía eólica ríos arriba captada por el rotor.

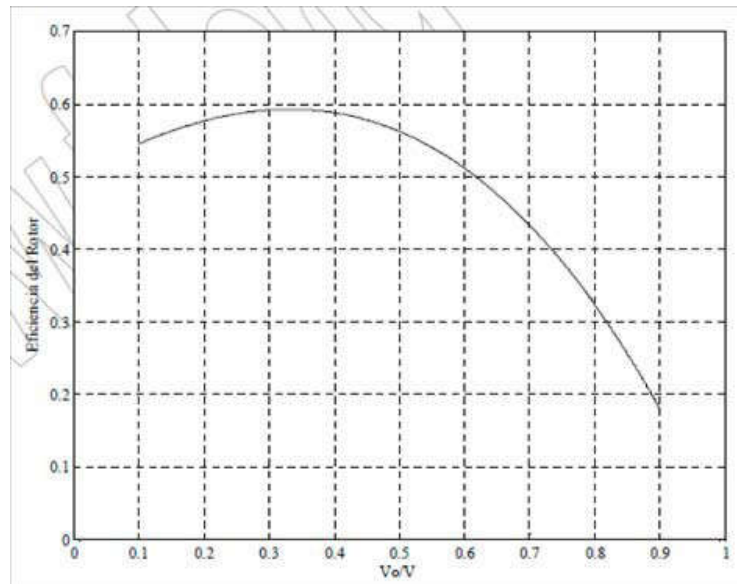

Imagen 3: "Eficiencia del rotor versus la relación Vo/V". Fuente [6]

En la imagen 3, se observa que $\mathrm{Cp}$ solo puede tener un valor máximo para la función pues este depende de la relación velocidades del viento aguas arriba y aguas abajo (Vo/V). Este máximo valor es de 0.59 (Postulado por Betz, cuando la relación $V o / V$ es $1 / 3$ ), en la práctica la potencia máxima extraíble por un aerogenerador oscila entre: 20 y $50 \%$. Por tanto la máxima potencia extraída del viento, cuando se tiene que la velocidad del viento río abajo es un $1 / 3$ del viento rio arriba es [6]:

$$
P_{\text {cmax }}=0.59 \frac{1}{2} \rho A V^{3}
$$

Ecuación 11: "Máxima potencia extraída del viento"

\section{DISTRIBUCIÓN DE VELOCIDADES DEL VIENTO MEIDANTE LA LEY DE WEIBULL}

La ley de Weybull nos permite determinar la distribución de velocidades del viento durante un determinado periodo de tiempo (recomendable no menor a 5 años). La función de densidad de probabilidad $p(v)$ correspondiente a la ley de Weibull es del tipo de dos parámetros ( $\mathrm{A}$ y $\mathrm{k}$ ) y está dada por la siguiente ecuación [7]:

$$
p(v)=\frac{k}{A}\left(\frac{v}{A}\right)^{k-1} e^{-\left(\frac{V}{a}\right)^{k}}
$$

Ecuación 12: "Función de densidad de probabilidad"

Dónde:

- v: velocidad del viento $[\mathrm{m} / \mathrm{s}]$,

- $p(v)$ : función densidad de probabilidad de Weibull,

- A: factor de escala [m/s], valor que suele ser próximo a la velocidad media (también expresado por la letra c).

Revista TECNIA Vol.27 N² Julio-Diciembre 2017 
- k: factor de forma que caracteriza la asimetría o sesgo de la función probabilidad.

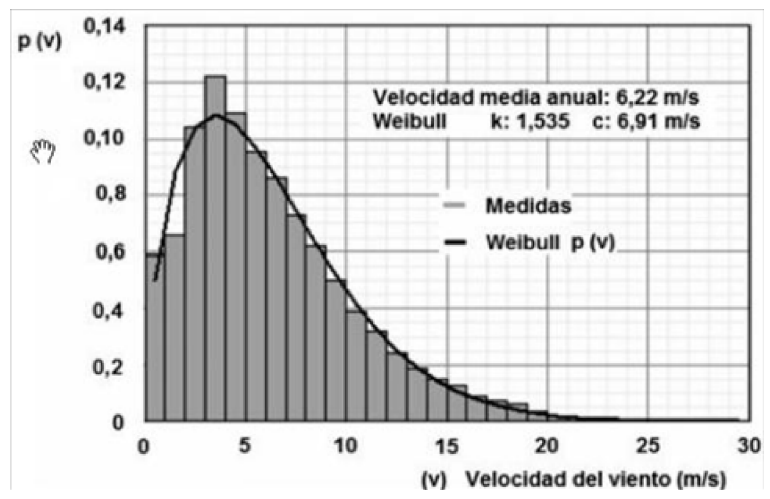

Imagen 4: "Distribución de la velocidad del viento durante un año (distribución de frecuencias relativas y la ley de Weibull ajustada). Fuente [5]

La siguiente imagen muestra un histograma anual de las velocidades del viento en donde las ordenadas representan las horas anuales (frecuencias relativas acumuladas), en lugar de frecuencias relativas (como en la imagen anterior).

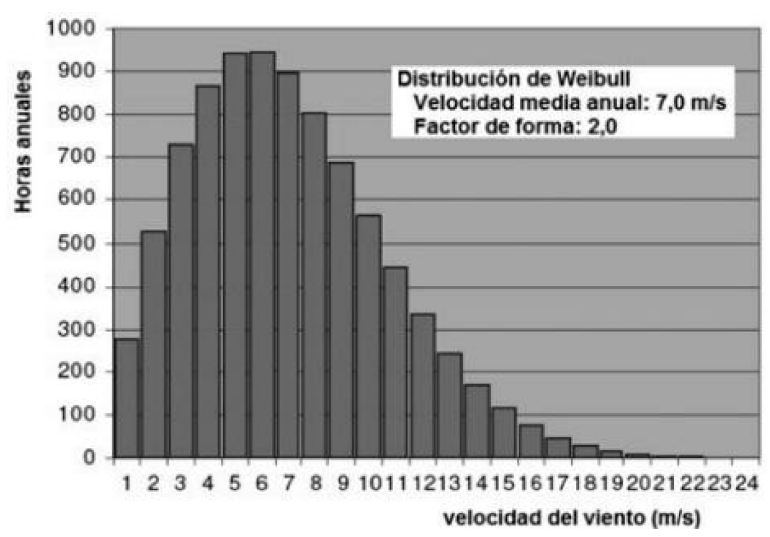

Imagen 5: "Histograma en horas de la velocidad del viento para 8760 horas (1 año)". Fuente [5]

Un caso particular es la "distribución de Rayleigh" cuando se tiene $k=2$, lo cual es un valor típico en algunas localidades. Valores como 2.5 o 3 indican que las variaciones horarias promedio de la velocidad del viento es pequeña en comparación con la variación promedio anual. En cambio valor como 1.5 o 1.2 nos indican una gran variación sobre el promedio. La siguiente imagen nos permite apreciar la influencia del parámetro "k" en la forma de la curva.

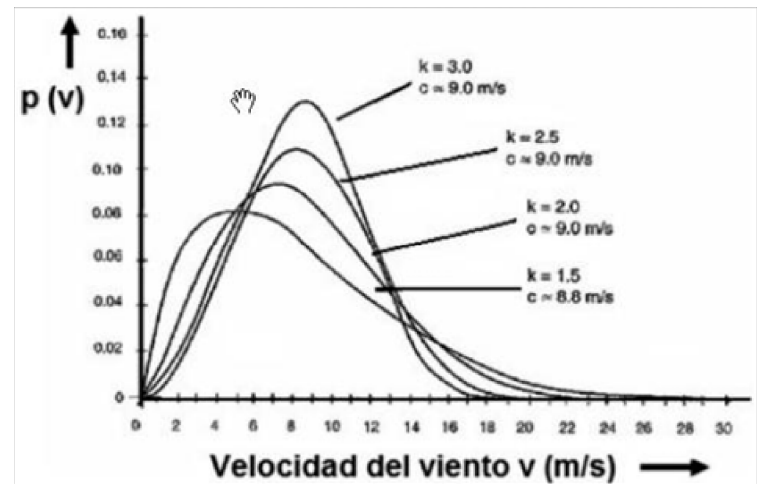

Imagen 6: "Densidad de probabilidad $p(v)$ de la velocidad del viento según la ley de Weibull para distintos valores de k". Fuente [5]

\subsection{Distribución de probabilidad acumulada de Weibull para velocidad del viento:}

Esta función representa la frecuencia acumulada para cada valor de velocidad del viento. [8]

$$
F(v)=1-e^{-\left(\frac{v}{A}\right)^{k}}
$$

Ecuación 13: "Función de distribución acumulada"

Dónde:

- F(v): Función de distribución de probabilidad acumulada de Weibull para velocidad del viento.

- v: velocidad del viento [m/s].

- A: factor de escala [m/s], valor que suele ser próximo a la velocidad media (también expresado por la letra c).

- k: factor de forma que caracteriza la asimetría o sesgo de la función probabilidad.

\subsection{Tratamiento estadístico para determinar " $\mathrm{K}$ " y “A"}

Para calcular los valores " $K$ " y " $A$ " se utilizará el método estadístico de regresión lineal, aplicándolo a la ecuación de distribución de probabilidad acumulada de Weibull.

$$
\begin{gathered}
\operatorname{Ln}(-\operatorname{Ln}(1-F(v)))=k \operatorname{Ln}(v)-k \operatorname{Ln}(A) \\
Y=m X+b
\end{gathered}
$$

Donde:

- Función lineal: $Y=\operatorname{Ln}(-\operatorname{Ln}(1-F(v)))$

- Variable en función de la velocidad del viento: $X$ $=\operatorname{Ln}(v)$

- Pendiente de la línea recta: $m=k$

- Constante: $\mathrm{b}=-\mathrm{kLn}(\mathrm{A})$ 
Luego de aplicar el método de regresión lineal, para calcular el valor de " $K$ " se debe hallar el valor de la pendiente de la línea recta que se gráfica, el resultado será igual al valor numérico de "K".

$Y$ para calcular el valor de "A" se debe hallar el valor de " $b$ " que al ser una constante se obtiene de la resta " $Y-m X$ " en cualquier punto, luego el valor de " $A$ " será igual al exponencial del cociente entre " $b$ " $y$ "-K".

\section{ENERGÍA ANUALMENTE GENERADA Y FACTOR DE CARGA DE UN PARQUE EÓLICO}

Las frecuencias relativas acumuladas obtenidas (ya sea mediante Excel u algún software como el Homer Pro) a partir del tratamiento estadístico de los datos registrados en la zona (recomendable no menor a 5 años) y a la altura de estudio nos determina la cantidad de horas mayor o igual a una velocidad de viento; esta y la curva de potencia del aerogenerador nos determina la cantidad de energía eléctrica producida anualmente.

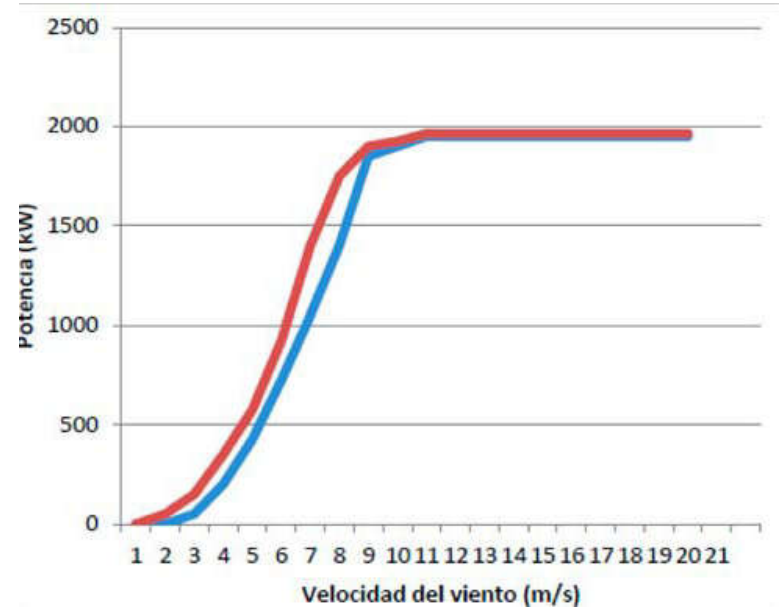

Imagen 7: "Comparación de la curva de potencia para 2 modelos de aerogeneradores"

La siguiente ecuación determina la energía entregada anualmente [9]:

$$
E E P=n^{\circ} \text { de horas }(v) * P(v)
$$

Ecuación 14: "Energía generada anualmente"

- Donde $p(v)$ : potencia generada a una velocidad de viento, según el modelo de aerogenerador seleccionado.

El valor obtenido mediante la ecuación anterior nos permite determinar las horas equivalentes (horas que tardaría en producir esa energía el aerogenerador trabajando a plena carga) [9]:

$$
H_{e q}=\frac{E E P(k W h)}{P_{n}(k W)}
$$

Ecuación 15: "Horas equivalentes"

Según [4] el factor de carga se define como la relación entre la energía producida por un aerogenerador, o un parque eólico, durante un periodo de tiempo y la energía que se hubiera generado durante ese periodo de tiempo si se hubiera trabajado a plena carga [9]:

$$
\begin{gathered}
F_{c}=\frac{E E P(k W h)}{P_{n}(k W) * 8760(h)}=\frac{H_{e q}}{8760} \\
\text { Ecuación 16: "Factor de carga }
\end{gathered}
$$

\section{ROSA DE VIENTOS}

Consiste en un diagrama polar en el cual se definen para diferentes direcciones o rumbos distintos valores relacionados con la velocidad y dirección del viento. El número de rumbos, cuyos valores principales se hacen corresponder con los puntos cardinales, suele ser 8,12 o 16. El porcentaje de calmas se indica en el centro del diagrama. viento:

Se puede dibujar diferentes tipos de rosa de

- Porcentaje del tiempo total que el viento sopla procedente de una determinada dirección (Imagen 8). Así, en esta figura se puede ver que la dirección dominante es la NO, de la cual sopla el viento el $23.64 \%$ de las horas anuales.

- $\quad$ Representación para cada dirección de porcentajes de tiempo total para los que la velocidad se mantiene en determinados intervalos de tiempo.

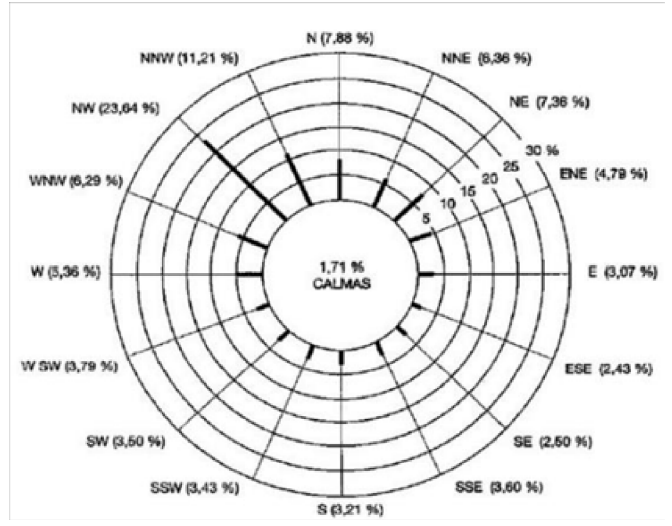

Imagen 8: "Rosa de direcciones de frecuencias porcentuales. Porcentaje de tiempo anual durante el tiempo que el viento procede de una dirección". Fuente [5] 


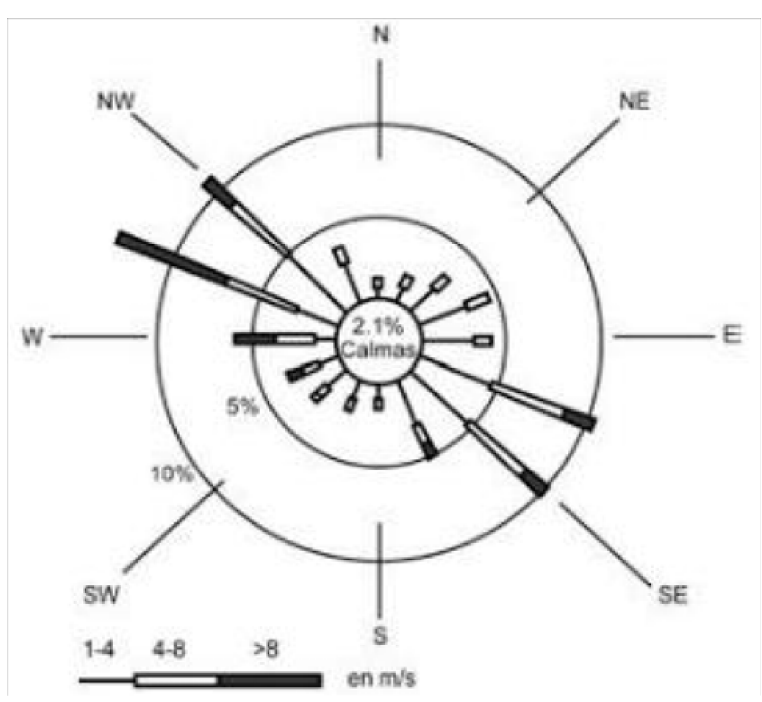

Imagen 9: "Frecuencia porcentuales de dirección para tres intervalos de las velocidades media". Fuente [5]

\section{RUGOSIDAD DEL TERRENO Y VARIACIÓN DE LA VELOCIDAD CON LA ALTURA}

Según [7] para caracterizar las condiciones de un terreno, se define el parámetro, denominado "longitud de rugosidad", como la altura respecto al nivel del suelo expresado en metros, donde la velocidad del viento es nula. En función del valor de se puede clasificar el terreno en clases de rugosidad. La relación entre la clase de rugosidad y la longitud de rugosidad viene dada por:

$$
z_{0} \leq 0.03 m \quad \text { Clase }=\frac{\ln \left(5000 z_{0}\right)}{\ln 150}
$$

Ecuación 17: "Rugosidad para Zo < 0.03 m"

$$
z_{0} \leq 0.03 m \quad \text { Clase }=\frac{\ln \left(\frac{1000}{9} z_{0}\right)}{\ln \left(\frac{10}{3}\right)}
$$

Ecuación 18: "Rugosidad para Zo>0.03 m"

En el cálculo de la variación de la velocidad con la altura se distingue dos situaciones [7]:

a) Variación con la altura de la velocidad del viento medio o estacionario.

Es el modelo más utilizado basado en la hipótesis de una atmósfera neutra, viene dada por la expresión:

$$
v^{\prime}=v \frac{\ln \left(\frac{z^{\prime}}{z_{0}}\right)}{\ln \left(\frac{z^{\prime}}{z_{0}}\right)}
$$

Dónde:
- $\quad v^{\prime}$ : velocidad del viento a una cierta altura sobre el nivel del suelo

- $\quad$ v: Velocidad de referencia, dato ya conocido $(\mathrm{m} / \mathrm{s})$

- $\quad z^{\prime}$ : Altura sobre el nivel del suelo para una velocidad deseada

- $\quad$ z: altura de referencia, es decir, la altura a la que conocemos la velocidad de viento exacta

zo: longitud de rugosidad en la dirección de viento actual.

La Tabla 4 muestra la clasificación de los terrenos en función de la longitud de rugosidad. Además, se incluye el índice de energía que permite estimar la pérdida potencial de energía eólica disponible en función de las características del terreno (superficies de agua, pistas de hormigón, campos abiertos sin cercados, campos con algunas casas, etc.)

\begin{tabular}{|c|c|c|c|}
\hline $\begin{array}{l}\text { Clase de } \\
\text { rugosidad }\end{array}$ & $\begin{array}{l}\text { Longitud de } \\
\text { rugosidad } \\
\left(\mathrm{z}_{\mathrm{o}}\right)(\mathrm{m})\end{array}$ & $\begin{array}{l}\text { Índice de } \\
\text { energía } \\
(\%)\end{array}$ & Tipo de terreno \\
\hline 0 & 0.0002 & 100 & $\begin{array}{l}\text { Superficie de agua. } \\
\text { Terreno descubierto, } \\
\text { superficie lisa. }\end{array}$ \\
\hline 0.5 & 0.0024 & 73 & $\begin{array}{l}\text { Pistas de hormigón (aero- } \\
\text { puertos), césped. }\end{array}$ \\
\hline 1 & 0.0030 & 52 & $\begin{array}{l}\text { Campos abiertos sin cerca- } \\
\text { dos ni setos. Edificios muy } \\
\text { dispersos. Colinas suave- } \\
\text { mente redondeadas. }\end{array}$ \\
\hline 1.5 & 0.055 & 45 & $\begin{array}{l}\text { Campo con algunas casas } \\
\text { y arbolado de hasta } 8 \mathrm{~m} \\
\text { situado como mínimo a } \\
1250 \mathrm{~m} \text {. }\end{array}$ \\
\hline 2 & 0.1 & 39 & $\begin{array}{c}\text { Campo con algunas casas } \\
\text { y arbolado de hasta } 8 \mathrm{~m} \\
\text { situado como mínimo a } \\
500 \mathrm{~m} \text {. }\end{array}$ \\
\hline 2.5 & 0.2 & 31 & $\begin{array}{l}\text { Campo con muchas casas } \\
\text { y arbolado de hasta } 8 \mathrm{~m} \\
\text { situado a } 250 \mathrm{~m} .\end{array}$ \\
\hline 3 & 0.4 & 24 & $\begin{array}{l}\text { Pueblos, bosques y } \\
\text { terreno }\end{array}$ \\
\hline 3.5 & 0.8 & 18 & $\begin{array}{c}\text { Ciudades con edificios y } \\
\text { terreno }\end{array}$ \\
\hline 4 & 1.6 & 13 & $\begin{array}{l}\text { Grandes ciudades con edi- } \\
\text { ficios muy elevados }\end{array}$ \\
\hline
\end{tabular}

Tabla 2: "Clases y longitudes de rugosidades". Fuente [7]

b) Variación con la altura de la velocidad media del viento a largo plazo.

En esta situación el modelo de cálculo más usado se basa en admitir una ley exponencial de Hellmann para la variación de la velocidad con la altura según la expresión: 


$$
\frac{v^{\prime}}{v}=\left(\frac{z^{\prime}}{z}\right) \alpha
$$

- Dónde: a es el exponente de Hellmann que varía según la rugosidad.

La siguiente tabla muestra los valores de exponente de Hellmann [7]:

Tabla 3: "Valores del exponente de Hellmann en función de la rugosidad del terreno"

\begin{tabular}{|l|c|}
\hline & $\begin{array}{c}\text { Coeficiente de } \\
\text { Hellmann }(\alpha)\end{array}$ \\
\hline Lugares Ilanos con hielo o hierba & $0.08-0.12$ \\
\hline Lugares Ilanoss (mar, costa) & 0.14 \\
\hline Terrenos poco accidentados & $0.13-0.16$ \\
\hline Zonas turísticas & 0.2 \\
\hline Terrenos accidentados o bosques & $0.2-0.26$ \\
\hline Terrenos muy accidentados y ciudades & $0.25-0.4$ \\
\hline
\end{tabular}

\section{CASO TÍPICO DISTRITO DE PIMENTEL}

\subsection{Parámetros técnicos del parque fotovoltaico}

Para el presente artículo se evalúa la siguiente zona de Pimentel:

- Latitud: $6^{\circ} 51^{\prime} 30.79^{\prime \prime S}$

- Longitud: $79^{\circ} 56^{\prime} 4.75^{\prime \prime O}$

- $\quad$ Altura sobre el nivel del mar:

- Área de Estudio: 45 hectáreas

- Potencia nominal de cada aerogenerador: 3MW

- Diámetro de las aspas: 50 metros

- Velocidad promedio anual: $9.28 \mathrm{~m} / \mathrm{s}$

- Altura al buje: $100 \mathrm{~m}$

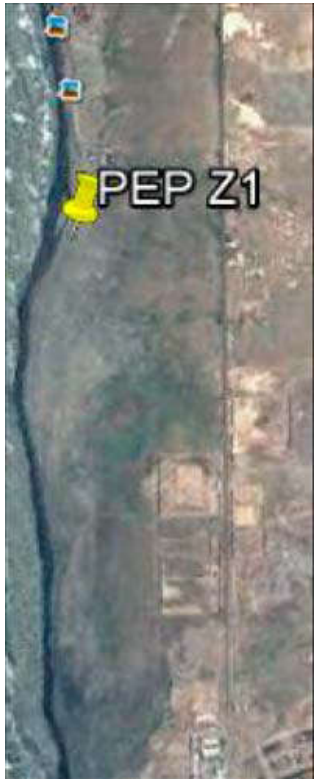

Imagen 10: Ubicación

Para analizar el potencial eólico del área seleccionada ( 45 hectáreas) se hace uso de la base de datos estadística proporcionada en el "atlas eólico 2016-MINEM".

Tabla 4: Producción Anual. Fuente: MINEM

\begin{tabular}{|c|c|c|c|}
\hline $\begin{array}{l}\text { Rango } \\
(\mathrm{m} / \mathrm{s})\end{array}$ & Horas (h) & $\begin{array}{l}\text { Potencia } \\
(\mathrm{kW})\end{array}$ & $\begin{array}{l}\text { Producción } \\
\text { (MWh/año) }\end{array}$ \\
\hline 3 & 175 & 0 & 0 \\
\hline 3.5 & 88 & 0 & 0 \\
\hline 4 & 88 & 47 & 4 \\
\hline 4.5 & 175 & 111 & 19 \\
\hline 5 & 175 & 190 & 33 \\
\hline 5.5 & 263 & 283 & 74 \\
\hline 6 & 263 & 391 & 103 \\
\hline 6.5 & 263 & 518 & 136 \\
\hline 7 & 350 & 666 & 233 \\
\hline 7.5 & 350 & 842 & 295 \\
\hline 8 & 438 & 1038 & 455 \\
\hline 8.5 & 438 & 1254 & 549 \\
\hline 9 & 438 & 1486 & 651 \\
\hline 9.5 & 438 & 1732 & 759 \\
\hline 10 & 438 & 1994 & 873 \\
\hline 10.5 & 438 & 2257 & 989 \\
\hline 11 & 438 & 2495 & 1093 \\
\hline 11.5 & 438 & 2694 & 1180 \\
\hline 12 & 438 & 2825 & 1237 \\
\hline 12.5 & 350 & 2907 & 1019 \\
\hline 13 & 350 & 2953 & 1035 \\
\hline 13.5 & 350 & 2984 & 1046 \\
\hline 14 & 263 & 2994 & 787 \\
\hline 14.5 & 263 & 2999 & 788 \\
\hline 15 & 175 & 3000 & 526 \\
\hline$>15$ & 701 & 3000 & 2102 \\
\hline
\end{tabular}




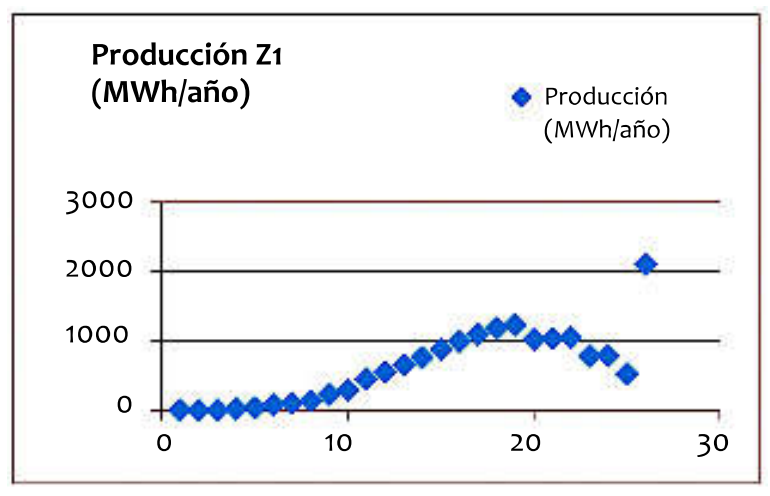

Imagen 11: Producción según velocidades específicas

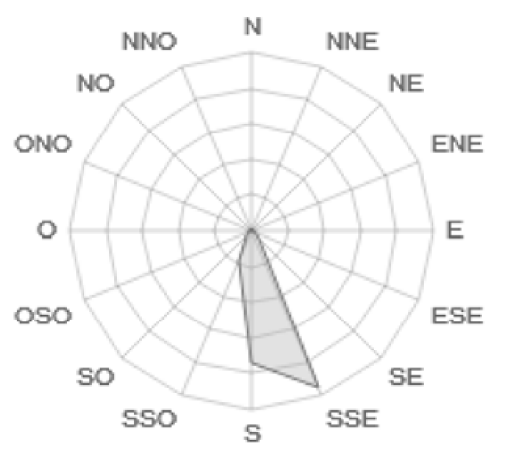

Imagen 12: Rosa de vientos

Mediante estos datos proporcionados por el MINEM (teniendo en cuenta el tipo de aerogenerador utilizado para evaluar el recurso eólico) se obtienen los siguientes valores:

\section{Tabla 5:}

\begin{tabular}{|l|c|c|c|c|c|}
\hline & $\begin{array}{c}\text { RUGOSI- } \\
\text { DAD }(\mathbf{m})\end{array}$ & $\begin{array}{c}\text { POTENCIA } \\
\text { DE INSTALA- } \\
\text { CIÓN }(\mathrm{kW})\end{array}$ & $\begin{array}{c}\text { ENERGíA } \\
\text { INYECTADA } \\
(\mathrm{kWh})\end{array}$ & $\begin{array}{c}\text { HORAS } \\
\text { EQUIVALENTES }(\mathrm{h})\end{array}$ & $\begin{array}{c}\text { FACTOR } \\
\text { DE CARGA }\end{array}$ \\
\hline ZONA 1 & 0.01 & 21000 & 111902000 & 5328.666667 & 0.608295282 \\
\hline
\end{tabular}

\subsection{Rentabilidad del proyecto}

La siguiente tabla muestra los parámetros económicos considerados para el parque FV de 5.4 MW.

Tabla 6: “Inversión Inicial”. Fuente Elaboración propia

\begin{tabular}{|c|c|}
\cline { 2 - 2 } \multicolumn{1}{c|}{} & Valor \\
\hline Presupuesto (S/.) & $122,640,000$ \\
\hline Precio de venta kWh (cS/.kWh) & 0.198 \\
\hline Producción anual estimada (kWh/año) & 111902000 \\
\hline Años de estudio & 25 \\
\hline Inflación & $3.32 \%$ \\
\hline Tasas de descuento & $10 \%$ \\
\hline Mantenimiento y otros (S/.) & 5000000 \\
\hline
\end{tabular}

La tabla 5 muestra el flujo de ingresos y egresos anuales
Tabla 7: "Flujo de ingresos y egresos económicos"

\begin{tabular}{|c|c|c|c|c|c|c|}
\hline Año & $\begin{array}{c}\text { Precio } \\
(\mathrm{S} / . \mathrm{kWh})\end{array}$ & $\begin{array}{c}\text { Producción } \\
\text { estimada } \\
(\mathrm{kWh})\end{array}$ & $\begin{array}{c}\text { Ingresos } \\
(\mathrm{S} / .)\end{array}$ & $\begin{array}{l}\text { Manteni- } \\
\text { miento y } \\
\text { otros }(S / .)\end{array}$ & $\begin{array}{c}\text { Flujo } \\
\text { de caja } \\
(\mathrm{S} / .)\end{array}$ & \\
\hline 2017 & 0.198 & & & $5,000,000$ & $-87,400,000$ & -79454545.45 \\
\hline 2018 & 0.205 & $111,902,000$ & $22,892,195$ & $5,166,000$ & $17,726,195$ & 14649747.92 \\
\hline 2019 & 0.211 & $111,006,784$ & $23,462,998$ & $5,337,511$ & $18,125,487$ & 13617946.61 \\
\hline 2020 & 0.218 & $110,118,730$ & $24,048,034$ & $5,514,717$ & $18,533,317$ & 12658505.12 \\
\hline 2021 & 0.226 & $109,237,780$ & $24,647,657$ & $5,697,805$ & $18,949,852$ & 11766367.21 \\
\hline 2022 & 0.233 & $108,363,878$ & $25,262,232$ & $5,886,972$ & $19,375,259$ & 10936828.85 \\
\hline 2023 & 0.241 & $107,496,967$ & $25,892,130$ & $6,082,420$ & $19,809,711$ & 10165513.80 \\
\hline 2024 & 0.249 & $106,636,991$ & $26,537,735$ & $6,284,356$ & $20,253,379$ & 9448350.76 \\
\hline 2025 & 0.257 & $105,783,895$ & $27,199,438$ & $6,492,997$ & $20,706,441$ & 8781552.25 \\
\hline 2026 & 0.266 & $104,937,624$ & $27,877,639$ & $6,708,564$ & $21,169,075$ & 8161594.82 \\
\hline 2027 & 0.274 & $104,098,123$ & $28,572,751$ & $6,931,289$ & $21,641,463$ & 7585200.72 \\
\hline 2028 & 0.284 & $103,265,338$ & $29,285,196$ & $7,161,407$ & $22,123,789$ & 7049320.83 \\
\hline 2029 & 0.293 & $102,439,215$ & $30,015,405$ & $7,399,166$ & $22,616,239$ & 6551118.72 \\
\hline 2030 & 0.303 & $101,619,701$ & $30,763,821$ & $7,644,818$ & $23,119,002$ & 6087955.90 \\
\hline 2031 & 0.313 & $100,806,744$ & $31,530,898$ & $7,898,626$ & $23,632,272$ & 5657377.98 \\
\hline 2032 & 0.323 & $100,000,290$ & $32,317,102$ & $8,160,861$ & $24,156,241$ & 5257101.95 \\
\hline 2033 & 0.334 & $99,200,288$ & $33,122,910$ & $8,431,801$ & $24,691,108$ & 4885004.17 \\
\hline 2034 & 0.345 & $98,406,685$ & $33,948,810$ & $8,711,737$ & $25,237,073$ & 4539109.32 \\
\hline 2035 & 0.356 & $97,619,432$ & $34,795,303$ & $9,000,967$ & $25,794,336$ & 4217580.06 \\
\hline 2036 & 0.368 & $96,838,476$ & $35,662,903$ & $9,299,799$ & $26,363,104$ & 3918707.42 \\
\hline 2037 & 0.380 & $96,063,768$ & $36,552,136$ & $9,608,552$ & $26,943,584$ & 3640901.85 \\
\hline 2038 & 0.393 & $95,295,258$ & $37,463,541$ & $9,927,556$ & $27,535,985$ & 3382684.93 \\
\hline 2039 & 0.406 & $94,532,896$ & $38,397,672$ & $10,257,151$ & $28,140,521$ & 3142681.59 \\
\hline 2040 & 0.420 & $93,776,633$ & $39,355,095$ & $10,597,688$ & $28,757,407$ & 2919612.93 \\
\hline 2041 & 0.434 & $93,026,420$ & $40,336,391$ & $10,949,532$ & $29,386,859$ & 2712289.52 \\
\hline 2042 & 0.448 & $92,282,209$ & $41,342,155$ & $11,313,056$ & $30,029,099$ & 2519605.12 \\
\hline & & & & & VAN & 94798114.89 \\
\hline
\end{tabular}

Fuente: Elaboración propia

Obteniéndose los siguientes valores económicos:

-VAN: S/. 94798114.89 -TIR: 11.175\% -PR: 8 años 


\section{CONCLUSIONES}

a.- Podemos clasificar las capas atmosféricas según la diferencial de temperatura para dos distintas alturas (lo que se define como gradiente térmico) este valor será diferente según la zona de la atmosfera (diferentes alturas y presiones) y la hora del día (diferentes valores de radiación).

b.- Se establece como el límite de la potencia teórica aprovechable, según Betz como el $59 \%$ de la energia captada del viento, pero experimentalmente este valor oscila entre $20 \mathrm{y}$ $50 \%$.

c.- Para el estudio de factibilidad en un parque eólico lo recomendable es recolectar datos estadísticos para un periodo no menor de 5 años.

d.- La distribución de Weybull permite fácilmente obtener la cantidad de horas para ciertas velocidades de viento (frecuencias relativas acumuladas)

e.- El Factor de carga de un parque eólico durante un año (8760 horas) es un factor clave para la viabilidad de este tipo de proyecto ya permite identificar el aprovechamiento energético respecto de la potencia nominal del parque.

f.- Para la selección de la ubicación de un parque eólico es necesario conocer la rugosidad de los alrededores ya que si la orografía es variable, generara un mapa de rugosidad que descartara la viabilidad.

g.- A partir de los parámetros seleccionados para la evaluación económica se obtiene un VAN: S/.94 798 114.89, un TIR: 11.175 \% y un PR: 8 años. Lo que nos permite afirmar en primera instancia que es viable.

\section{BIBLIOGRAFÍA}

[1] Ministerio de Energía y Minas, «Decreto Legislativo $\mathrm{n}^{\circ} 1002$ Promoción de la inversión para la generación de electricidad con el uso de energías renovables", 2010.

[2] Dirección General de Electrificación rural, «Plan Nacional de Electrificación rural (PNER)», 2012

[3] La República, «Lambayeque: Realizarán estudios de factibilidad de Central Eólica José Quiñones», 2015.

[4] Comité de Operación Económica del Sistema Interconectado Nacional, «Estudio de la máxima capacidad de generación no convencional (eólica y solar) a ser instalada en el SEIN (CMGNC)", 2015.

[5] M. Villarubia «Ingeniería de la energia eólica», 2013.

[6] F. Gonzales Longatt, "Variación del viento y energía asociada», 2009.

[7] I. Dioses, «Evaluación del potencial eólico para un emplazamiento seleccionado en la región Piura utilizando $\mathrm{GH}$ Windfarmaer», Departamento de ingeniería mecánico-eléctrica, Facultad de ingeniería, Universidad de Piura, 2013.

[8] Ministerio de energía y minas, «Información sobre la curva de potencia para el Atlas eólico del Perú», 2016.

[9] Hernández Pérez L., "Diseño de un parque eólico de $50 \mathrm{MW}$ en el municipio de Salvacañete (cuenca). Consideraciones medioambientales y viabilidad económica", (2014).

[10] A. Molinero, "Proyecto de un parque eólico», Escuela técnica superior de ingeniería, Universidad Pontificia Comillas, 2009.

[11] P. Polanco, «Lineamientos para el desarrollo de parques eólicos en Guatemala», Escuela de ingeniería civil, Facultad de ingeniería, Universidad de San Carlos de Guatemala, 2012.

[12] D. Maldonado y D. De Jerónimo, «Ubicación de un parque de energía eólica en la costa ecuatoriana», Universidad San Francisco de Quito, 2008.

[13] R. García y V. Ortega, «Estudio de impacto ambiental del parque eólico "A Xunqueira"», Asturvent Energías S.L.

[14] J. Proboste, «Diseño y evaluación técnica económica de una planta eólica para la ciudad de Punta Arenas», Escuela de ingeniería mecánica, Facultad de ciencias de la ingeniería, Universidad Austral de Chile, 2007.

[15] J. Lucio, «Diseño de un parque eólico en el sector Pusuchisi alto del cantón Latacunga para el sistema de distribución de la empresa eléctrica Cotopaxi», Facultad de ingeniería eléctrica y electrónica, Escuela Politécnica Nacional, 2014.

[16] R. Bayón, L. Cebadera y R. Del Castillo, «Diseño de un parque eólico de $6 \mathrm{MW}$ en Malpica de Bergantiños, La Coruña», EOIEscuela de Negocios.

[17] A. Falces, «Planificación de parques eólicos mediante sistemas de información geográfica y algoritmos genéticos», Escuela técnica superior de ingeniería industrial, Universidad de la Rioja, 2015.

[18] M. Esteban, «Propuesta de una metodología para la implantación de parques eólicos offshore», Universidad Politécnica de Madrid, 2009. 